\title{
Computerized Determination of Growth Kinetic Curves and Doubling Times from Cells in Microculture
}

\author{
Herta Reile, Herbert Birnböck, Günther Bernhardt, Thilo Spruß, and Helmut Schönenberger \\ Institut für Pharmazie, Sonderforschungsbereich 234, Universität Regensburg, D-8400 Regensburg, Federal Republic of Germany
}

Received July 18, 1989

In this paper we describe the microcomputer-aided determination of cell proliferation kinetics and doubling times utilizing a crystal violet assay and a 3-(4,5-dimethylthiazol-2-yl)-2,5-diphenyl-2H-tetrazolium bromide assay in microtitration plates. The analysis of spectrophotometric data provides the doubling times at any time of incubation. Plots of doubling time versus time of incubation give reproducible information on the exact duration of the logarithmic growth phase. This method is applicable to anchorage-dependent as well as anchorage-independent cells when colorimetric or fluorometric data are accessible. ๑ 1990 Academic Press, Inc.

A great problem in the testing of new anticancer drugs by the cell culture technique is the marked genetic instability of tumor cells $(1,2)$ entailing an extreme clonal variation (3). This becomes evident with growing numbers of passages from a permanent change of parameters of cell proliferation like the duration of the lag phase and of the log period, the doubling time, and the saturation density (plateau level). For example, Reddel et al. (4) observed such a perpetual decrease of doubling time in subcultures of the human breast cancer cell line T-47-D.

Since drugs can show quite different inhibitory effects dependent of the growth parameters of a given tumor cell line, the knowledge of the growth parameters in each test series is of importance for the experimental reproducibility. Although it is generally postulated that the exposure of the tumor cells to the drug should occur in the phase of exponential growth, this demand often does not meet experimental verification.

Conventionally, mean doubling times are determined graphically from the mid log phase or calculated from initial and final cell numbers (5) counted with either a hemocytometer or an electronic particle counter which requires removing the cells from their substratum. Either method could lead to severe misinterpretation due to practical difficulties in distinguishing the lag phase from the phase of exponential growth.

In this publication we describe a microcomputer technique which allows the registration of growth curves of cells in monolayer cultures by large scale spectrophotometric measurement after crystal violet staining ${ }^{1}(6)$ or MTT incorporation (7-9) using 96-well microtitration plates. This method enables the overall growth curve especially the lag phase and the log period to be determined in a more exact way. As a consequence it is also possible to precisely assess the doubling time (TAU) at any time of the experiment. In our opinion a plot of TAU versus time $(t)$ provides the maximum information available from cell proliferation experiments.

\section{METHODS}

Chemicals. Reagents (A-grade purity) were obtained from Merck. Crystal violet and MTT were purchased from Serva; dimethyl sulfoxide (DMSO) spectrophotometric grade was obtained from Aldrich. Millipore-filtered water was used throughout.

Cell lines and culture conditions. MDA-MB-231 (ATCC No. HTB 26), a human adenocarcinoma of the breast, was maintained in McCoy's 5A medium (Boehringer) containing L-glutamine, $\mathrm{NaHCO}_{3}(2.3 \mathrm{~g} /$ liter $)$, gentamycin (50 mg/liter), and 10\% NCS (GIBCO) in 75$\mathrm{cm}^{2}$ flasks (Falcon Plastics 3023 ) as a monolayer.

The cells were serially passaged weekly following trypsinization using trypsin/EDTA (Boehringer).

$P 388 D_{1}$ (ATCC No. CCL 46), a murine lymphoid neoplasm, was grown in a suspension culture in Dulbecco's modified Eagle's medium (Seromed) containing glucose

\footnotetext{
${ }^{1}$ Abbreviations used: crystal violet, $N$-hexamethylpararosaniline; MTT, 3-(4,5-dimethylthiazol-2-yl)-2,5-diphenyl- $2 H$-tetrazolium bromide; DMSO, dimethyl sulfoxide; PBS, phosphate-buffered saline; $\mathrm{OD}$, optical density; PC, personal computer; NCS, newborn calf serum.
} 


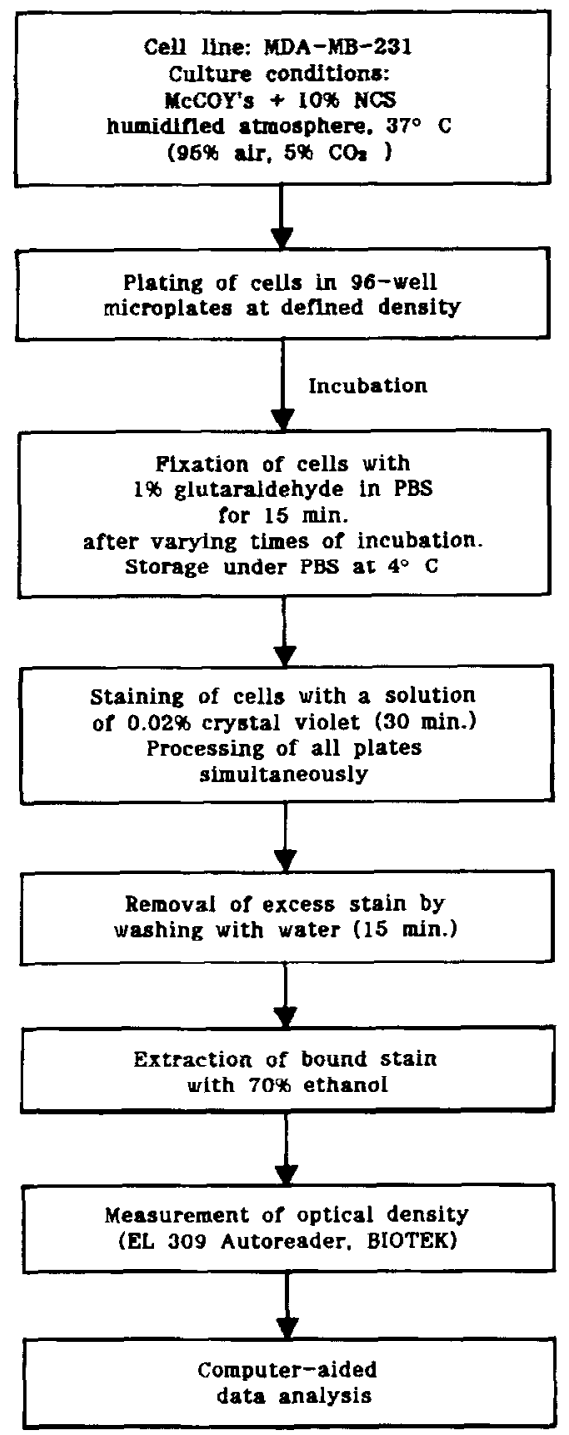

FLOW CHART 1. Registration of growth curves using the crystal violet dye procedure. Optical density reading at $578 \mathrm{~nm}$ gives improved sensitivity and reliability.

( $4.5 \mathrm{~g} /$ liter $), \mathrm{NaHCO}_{3}(3.7 \mathrm{~g} / \mathrm{liter})$, sodium pyruvate $(110$ $\mathrm{mg} / \mathrm{liter}$ ), gentamycin $(50 \mathrm{mg} / \mathrm{liter})$, and $10 \%$ horse serum (Boehringer) in $75-\mathrm{cm}^{2}$ culture flasks. The cells were passaged every $3-4$ days by 1:40 dilution with fresh medium.

Both cell lines were grown at $37^{\circ} \mathrm{C}$ in a humidified atmosphere of $95 \%$ air and $5 \%$ carbon dioxide.

Mycoplasma contamination was monitored by routine assay techniques (Hoechst 33.258) (10), and only mycoplasma-free cultures were used.

Registration of growth curves. The assay was carried out in flat-bottomed microtitration trays (Falcon Plastics 3075). Optical density was read using a Biotek EL 309 Autoreader (Tecnomara) at $578 \mathrm{~nm}$ (crystal violet) and $540 \mathrm{~nm}$ (MTT).
(I) $M D A-M B-231$ cells were processed as shown in Flow Chart 1 by modifying the crystal violet staining procedure described by Gillies et al. (6).

(II) The proliferation of $P 388 D_{1}$ cells was quantitated according to a microculture modification of the tetrazolium assay $(7-9,11)$ : MTT was prepared as a $1 \mathrm{mg} / \mathrm{ml}$ stock solution in medium and filtered through a $0.22-\mu \mathrm{m}$ filter to remove undissolved dye. At the times indicated in Fig. 1, $50 \mu \mathrm{l}$ of stock MTT solution was added to all wells, each containing $100 \mu \mathrm{l}$ of cell suspension. The plates were gently shaken and incubated at $37^{\circ} \mathrm{C}$ for another $1.5 \mathrm{~h}$. Supernatant removal was accomplished by carefully inverting and blotting the tray; no prior centrifugation was needed, since the macrophage-like cells sedimented and adhered to the substratum. One hundred microliters of DMSO was added to all wells to dissolve the formazan crystals.

Cytogenetic analysis. The cells were grown to about $50 \%$ confluence on microscopic slides. The slides were prepared as described elsewhere (12). So that spindle

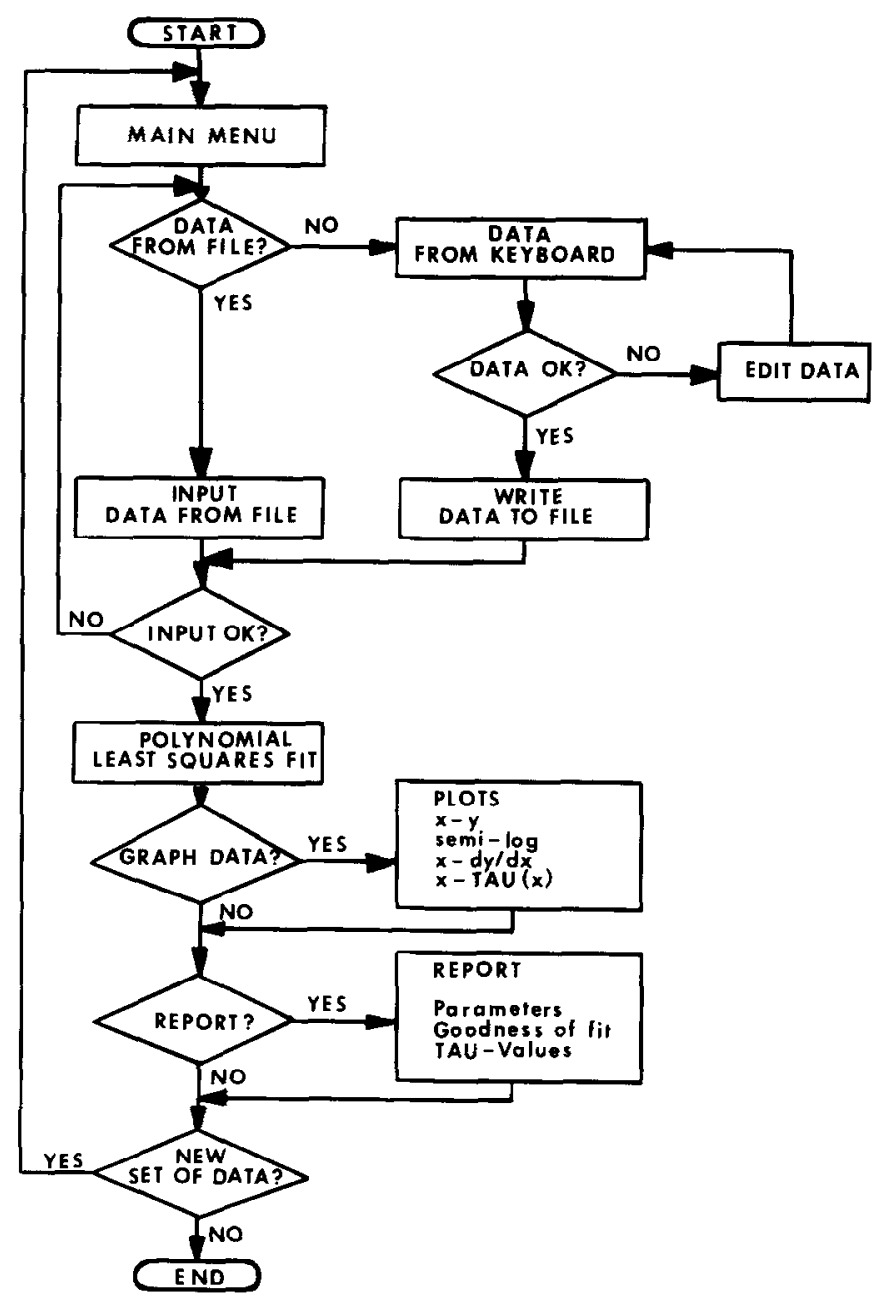

FLOW CHART 2. Analysis of spectrophotometric data. 

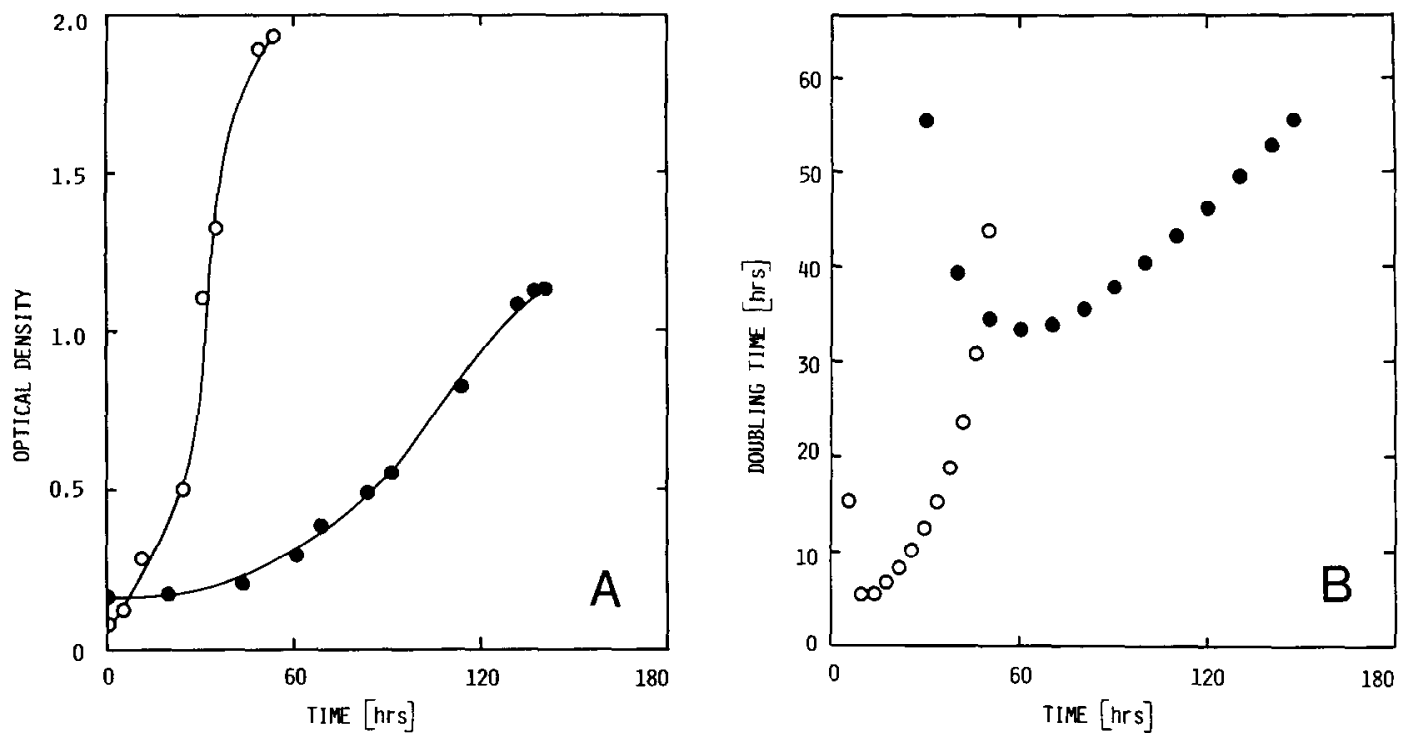

FIG. 1. (A) Colorimetric measurement of growth in microtitration plates. $(O) P 388 D_{1}$ leukemia cells from passage 28 (original passage unknown, numbered upon receipt from the ATCC) were seeded at a density of $8 \times 10^{4}$ cells $/ \mathrm{ml}$ (Coulter Counter $\mathrm{Z}_{\mathrm{B}} \mathrm{I}$ ). At the times indicated, MTT was added to all wells, and after another $1.5 \mathrm{~h}$ the plates were developed and read immediately at $540 \mathrm{~nm}$. ( $)$ The human breast cancer cell line $M D A-M B-231$ was plated in the 38 th passage from origin at a density of 12 cells per field of vision. The cells were seeded in $100 \mu l$ medium/well and were allowed to attach. Cell densities were estimated by counting 50 fields of vision using a Leitz Diavert phase-contrast microscope $(320 \times)$. At the times indicated, the cells were fixed with $1 \%$ glutaraldehyde in PBS for 15 min and stored under PBS at $4^{\circ} \mathrm{C}$. At the end of the experiment all trays were stained with crystal violet simultaneously. OD was measured after extraction of the dye with $70 \%$ ethanol at $578 \mathrm{~nm}$. (B) Plots of the corresponding doubling times, calculated from the data shown in A, versus time of incubation.

formation could be inhibited, the slide chambers were inoculated with Colcemid solution (Serva) to a final concentration of $0.04 \mu \mathrm{g} / \mathrm{ml}$ and incubated for $3 \mathrm{~h}$ at $37^{\circ} \mathrm{C}$. The medium was removed by suction and replaced with $0.075 \mathrm{M} \mathrm{KCl}$. After $30 \mathrm{~min}$ of incubation at $37^{\circ} \mathrm{C}$ an equal volume of cold, freshly made fixative (absolute methanol/glacial acetic acid, 3/1) was added. This hypotonic/ fixative mixture was removed immediately and replaced twice with ice-cold, fresh fixative. The slides were removed from the dish and air-dried.

The chromosomes were stained for $8 \mathrm{~min}$ with $10 \mathrm{ml}$ Giemsa plus $90 \mathrm{ml}$ of $0.025 \mathrm{M} \mathrm{KH}_{2} \mathrm{PO}_{4}, \mathrm{pH} 6.8$.

Computer processing. Readings from the EL 309 autoreader (Biotek) were transferred directly to an Olivetti M24 personal computer, using a program that saved the $O D$ values on a diskette. The program for data processing is graphed in Flow Chart 2.

Doubling time analysis. Curve fitting of experimental data is accomplished by a polynomial regression fit applying the least-squares method. Optical density is described as a function of time and data points are fitted to an $n$th order polynomial (usually $n=5$ ) represented by

$$
\begin{aligned}
\mathrm{OD}=f(t)=\sum a_{i} t^{i} ; & (i=0,1, \ldots, n) \\
& a_{i}=\text { regression coefficients. }
\end{aligned}
$$

This polynomial is defined in the time interval of points observed. Once the polynomial fit has been performed the doubling time TAU can easily be calculated at any time of the interval concerned provided that exponential growth is observed (13),

$$
\begin{aligned}
\mathrm{TAU} & =1 / \alpha \\
\alpha & =1 / \ln (2) \cdot d \ln (\mathrm{OD}) / d t \\
\mathrm{OD} & =\sum a_{i} t^{i},
\end{aligned}
$$

and hence

$$
\mathrm{TAU}=\ln (2) \cdot\left(\sum a_{i} t^{i}\right) /\left(\sum i \cdot a_{i} t^{i-1}\right) ; \quad(i=0,1, \ldots, n) .
$$

ABS (TAU) is plotted versus time. From this plot the time interval where exponential growth occurs and the corresponding doubling time can easily be determined. The software for evaluating cell kinetic data was developed in FORTRAN and PASCAL and implemented on an Olivetti M24 PC.

\section{RESULTS}

Figure 1 illustrates the growth curves and the corresponding doubling times at any time of incubation for an anchorage-dependent (MDA-MB-231) cell line and an anchorage-independent $\left(P 388 D_{1}\right)$ cell line. The curves were registered using two different staining tech- 


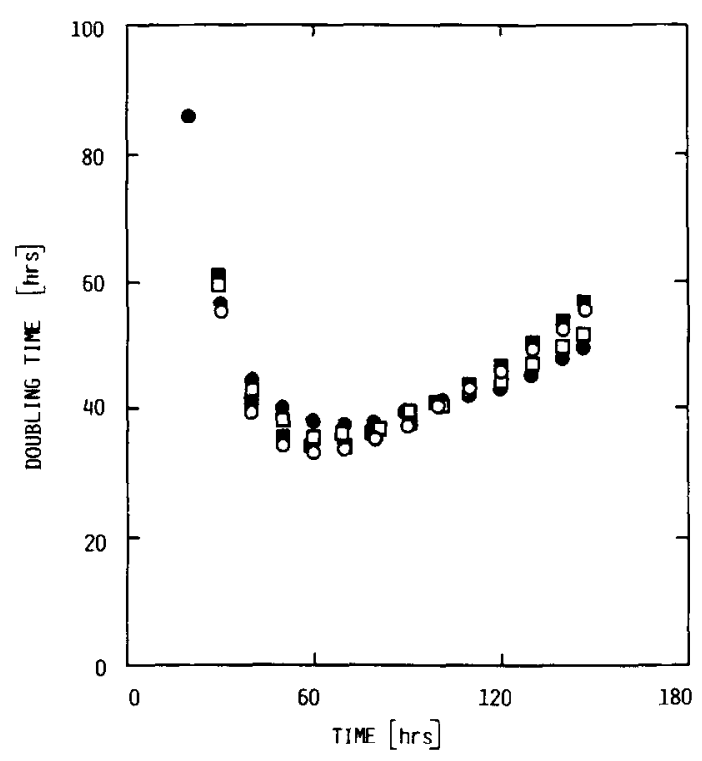

FIG. 2. Reproducibility of doubling time determination by the crystal violet assay. $M D A-M B-231$ cells were plated at an approximate density of 15 cells per field of vision (Leitz Diavert, $320 \times$ ). The calculated doubling times from four different experiments (all processed separately) are plotted versus time of incubation.

niques: crystal violet for $M D A-M B-231$ and MTT for $P 388 D_{1}$. Due to their macrophage-like properties (tendency to attach) no centrifugation step was required in the case of $P 388 D_{1}$ cells. A general application of the MTT procedure to anchorage-independent cells requires a centrifuge equipped with a rotor capable of accepting multiplates.

Under the given experimental conditions for both cell lines the logarithmic phase covers only a small fraction of the overall growth curve. In a plot, doubling time (TAU) versus time of incubation ( $t$ ) (Fig. 1B), exponential growth is characterized by a parallelism of the graphs with the $t$-axis. Although in Fig. $1 \mathrm{~A}$ the time points are not equidistant the calculated doubling times for the exponential growth phase are not significantly affected by closely spaced points at either end of the curve. A close scattering of data at both ends is required to separate the log phase from the lag and the plateau phases where cell proliferation becomes extremely slow resulting in infinite doubling times. Therefore only quantification of the $\log$ phase is of biological significance.

$M D A-M B-231$ grows exponentially for about two generations with a doubling time of $\approx 34 \mathrm{~h}$, whereas the log phase for $P 388 D_{1}$ at the given plating density is restricted to one doubling of the population.

By performing the doubling time calculations from four successive crystal violet processing procedures utilizing $M D A-M B-231$ cells in the same passage seeded at comparable plating densities, we encountered an interassay variation of $\leqslant 13 \%$ (Fig. 2).
The karyotype information presented in Fig. 3 gives an example for the dramatic variability of a well-defined cell line after prolonged culture in vitro following cryopreservation. Genotypic changes do not only reflect phenotypic characteristics like morphology, but may also alter cell physiology as well as cell proliferation, which directly affects doubling time (14).

Figure 4 illustrates the alterations in doubling time as a result of the number of passages. The doubling times of $M D A-M B-231$ cells maintained under constant culture conditions decreased constantly with prolonged time in culture. In the crystal violet assay the cells were seeded at comparable densities. The doubling times vary enormously over a wide range from $\approx 10 \mathrm{~h}$ for a late ( $85 \mathrm{th}$ ) to $\approx 38 \mathrm{~h}$ for a relatively early (35th) passage. The variation most probably arises from the selection of faster growing subclones from an initially heterogeneous population by frequent and incomplete trypsinization of the culture.

It is widely accepted that the initial cell number influences the proliferation kinetics of eukaryotic cells. This marked influence on doubling time is shown in Fig. 5.

\section{DISCUSSION}

While a variety of techniques $(6-10,15)$ including an enzyme immunoassay for estimating small cell numbers (16) have been described recently for the determination of parameters for cell proliferation, little interest was focused on the construction and analysis of growth curves in animal cell culture.

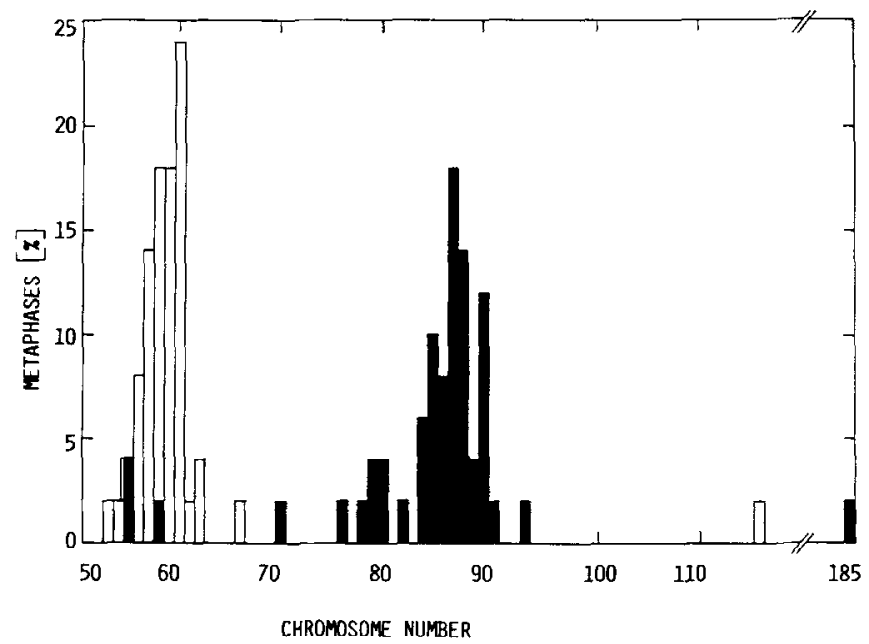

FIG. 3. Genetic instability of $M D A-M B-231$ cells. (ㅁ) 27th passage from origin, (匹) 35th passage from origin: The cells were cryopreserved in passage 28 for about 6 months and then passaged weekly, when rethawed. For each passage the chromsomes from 50 well-spread metaphase plates were counted. 
It has been common practice to assume that cells exhibit ideal exponential growth regardless of the diversities of experimental designs without further verification.

In our opinion the description of the overall growth characteristics with an exact determination of the lag/ log phases, the doubling time (TAU), and the plateau level is absolutely necessary. In addition to the characterization of cell lines (e.g., morphology, karyotype, receptor pattern, sensitivity to antitumor drugs) the description of the exponential growth phase is a prerequisite for any valuable experimental setup. Especially, the investigation of cell lines underlying extreme genetic variability, e.g., transformed cells and cancer cells, requires a precise description and verification of the parameters concerned. Due to time-consuming and difficult experimental procedures this is usually not performed.

The major disadvantage of the methods described so far $(5,17,18)$ is the production of mean values rather than exact values. Due to laborious working techniques only a few data points are generally obtained. This results in poor graphical estimates which make the separation of the lag/log/plateau phases of growth curves extremely difficult and unsatisfactory.

In this paper we describe a technique which offers a number of advantages compared with conventional methods. This inexpensive, rapid, and technically easy microtitration assay gives access to large sets of data thus providing results of high statistical significance. The equipment necessary (microplate reader, $\mathrm{PC}$ ) is available in almost every analytical laboratory today.

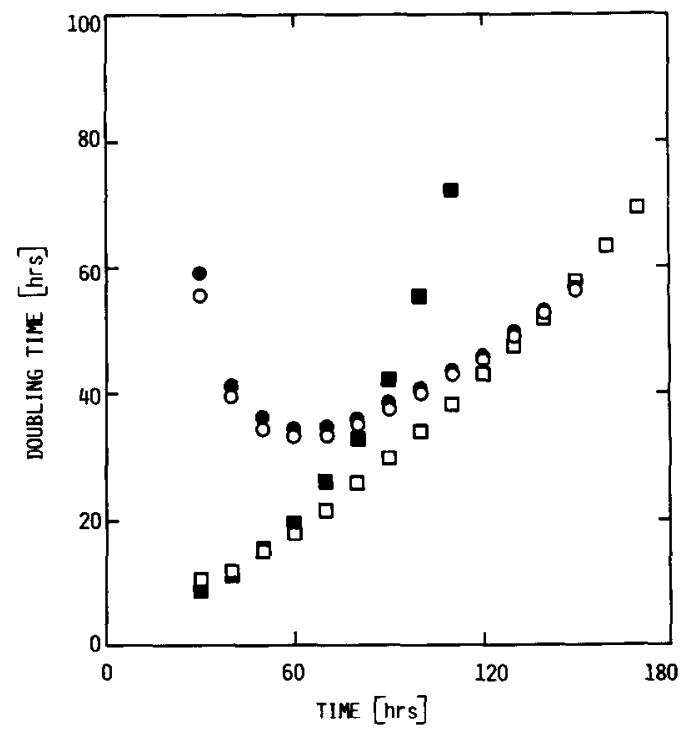

FIG. 4. Doubling time as a function of passage using $M D A-M B-231$ cells in the $(O)$ 35th, $(\bullet) 39$ th, and $(\square, \square)$ 85th passage. ( $\square)$ The cells were plated at double density (ca. 30 cells per field of vision, Leitz Diavert, 320 X).

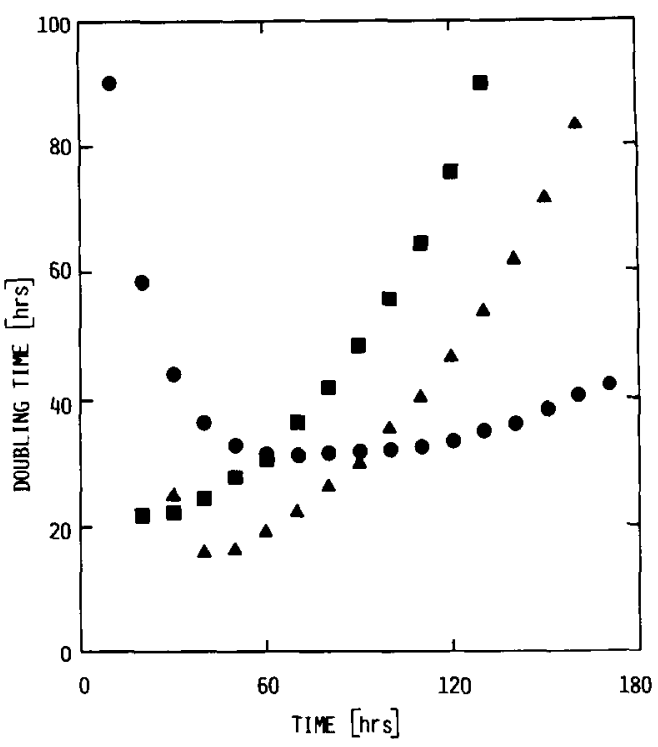

FIG. 5. Doubling time as a function of initial cell number. $M D A-$ $M B-231$ cells were plated in passage 39 at different densities: (๑) 10 , (A) 16 , and (D) 36 cells per field of vision (Leitz Diavert, $320 \times$ ).

Because of the mathematical representation of the data an exact determination of the log period is guaranteed. TAU as a function of time clearly reveals the duration of the lag and plateau phases. This approach overcomes the major drawback of graphical methods which only give a poor estimate of cell kinetics. This method offers universal applicability to biological investigations whenever growth kinetics must be monitored. Its use is by no way restricted to colorimetric measurement. Its application can also be extended to turbidimetric, fluorometric, radiochemical, etc., monitoring techniques. The only prerequisite is that a large number of data which can be digitized are available.

\section{ACKNOWLEDGMENTS}

This work was supported by the Deutsche Forschungsgemeinschaft and the "Matthias Lackas-Stiftung für Krebsforschung." Thanks are also due to the Fonds der Chemischen Industrie for financial support. The helpful assistance of F. Birk in solving data transfer problems is gratefully acknowledged.

\section{REFERENCES}

1. Whang-Peng, J., Lee, E. C., Kao-Shan, C.-S., Seibert, K., and Lippman, M. (1983) J. Natl. Cancer Inst, 71, 687-695.

2. Yunis, J. J. (1983) Science 221, 227-236.

3. Seibert, K., Shafie, S. M., Triche, T. J., Whang-Peng, J. J., O'Brien, S. J., Toney, J. H., Huff, K. K., and Lippman, M. E. (1983) Cancer Res. 43, 2223-2239.

4. Reddel, R. R., Alexander, I. E., Koga, M., Shine, J., and Sutherland, R. L. (1988) Cancer Res. 48, 4340-4347.

5. Reddel, R. R., Murphy, L. C., Hall, R. E., and Sutherland, R. L. (1985) Cancer Res. 45, 1525-1531. 
6. Gillies, R. .J., Didier, N., and Denton, M. (1986) Anal. Biochem. 159, 109-113.

7. Mosmann, T. (1983) J. Immunol. Methods 65, 55-63.

8. Denizot, F., and Lang, R. (1986) J. Immunol. Methods 89, 271277.

9. Alley, M. C., Scudiero, D. A., Monks, A., Hursey, M. L., Czerwinski, M. J., Fine, D. L., Abbott, B. J., Mayo, J. G., Shoemaker, R. H., and Boyd, M. R. (1988) Cancer Res, 48, 589-601.

10. Peters, J. H., Baumgarten, H., and Schulze, M. (1985) Monoklonale Antikörper: Herstellung und Charakterisierung, pp. 94-101, Springer, Berlin/Heidelberg/New York/Tokyo.

11. Carmichael, J., DeGraff, W. G., Gazdar, A. F., Minna, J. D., and Mitchell, J. B. (1987) Cancer Res. 47, 936-942.
12. Rooney, D. F., and Czepulkowski B. H. (1986) in Human Cytogenetics, A Practical Approach (Rooney, D. E., and Czepulkowski B. H., Eds.), p. 10, IRL Press, Oxford/Washington, DC.

13. Griffiths, B. (1986) in Animal Cell Culture, Practical Approach (Freshney, R. I., Ed.), p. 38, IRL Press, Oxford/Washington, DC.

14. Osborne, C. K., Hobbs, K., and Trent, J. M. (1987) Breast Cancer Res. Treat. 9, 111-121.

15. Romijn, J. C., Verkoelen, C. F., and Schroeder, F. H. (1988) Prostate 12, 99-110.

16. Faraji-Shadan, F., and Bowman, P. D. (1989) Anal. Biochem. 177, 259-262.

17. Roper, P. R., and Drewinko, B. (1976) Cancer Res. 36, 2182-2188.

18. Leonessa, F., Coialbu, T., and Toma, S. (1986) Anticancer Res. 6, $1291-1296$. 